\title{
Total Umwelten Create Shared Meaning the Emergent Properties of Animal Groups as a Result of Social Signalling
}

\author{
Amelia Lewis ${ }^{1}$ (iD \\ Received: 9 April 2020 / Accepted: 25 October 2020 / Published online: 2 November 2020 \\ (C) The Author(s) 2020
}

\begin{abstract}
In this paper, I discuss the concept of 'shared meaning', and the relationship between a shared understanding of signs within an animal social group and the Umwelten of individuals within the group. I explore the concept of the 'Total Umwelt', as described by Tønnesen, (2003), and use examples from the traditional ethology literature to demonstrate how semiotic principles can not only be applied, but underpin the observations made in animal social biology. Traditionally, neo-Darwinian theories of evolution concentrate on 'fitness' or an organism's capacity to survive and reproduce in its own environmental niche. However, this process also relies on underlying signs and sign processes, which are often over-looked in traditional ethology and behavioural ecology. Biosemiotics, however, places the emphasis on sign process, with signs and signals comprising a semiosphere. Significantly, whilst the semiosphere is formulated as physical phenomena, specifically energetic and material signs which can be detected and transmitted as signals from one individual to another, it is the Umwelten of living organisms which give those signals meaning. Further, two or more Umwelten can merge, giving rise to a 'Total Umwelt', which facilitates shared meaning of signs between two or more individuals. Across and within generations, this gives rise to cultural interpretation of signs within populations. I argue this is the fundamental basis for emergent group properties in social species, or indeed in solitary living species where individuals interact to mate, defend territories or resources, or in raising altricial young. I therefore discuss a fusion of traditional behavioural ecology- based theory with semiotics, to examine the phenomenon of 'shared meaning' in animal social groups.
\end{abstract}

Keywords Umwelt - Semiosphere - Emergent behaviour · Shared meaning - Social group · Communication

Amelia Lewis

Alewis08@qub.ac.uk

1 School of Biological Sciences, Queen's University, Belfast, Belfast, Northern Ireland, UK 


\section{Introduction}

The Umwelt, as first proposed by Jakob von Uexküll, describes the perceptual and effector domain of an individual organism, within the physical world. The Umwelt relies upon signs which are imperative in allowing an individual to perceive and process data about the physical environment it inhabits, at both a whole organism and cellular level (Ginn 2014; Hoffmeyer, 2008a, b). However, this means that an organism's experiences of the physical world are restricted by both its sensory apparatus and its ability to disperse into different environments. An individual's perception of the world is thus constrained by physical parameters. For example, a fish has no experience of terrestrial ecosystems, and its perceptual range is limited to the aquatic environment. Further, signals which are transmitted in the terrestrial environment are distorted on passing into the aquatic domain. Light is refracted, and acoustic signals, travelling through water rather than air, have their spectral characteristics altered by the new medium. This means that beyond its own habitat niche, a fish can have only very limited knowledge of terrestrial environments. Furthermore, what data it does receive from land will be difficult to process, as an aquascape is the only schema a fish knows as a basis for comparison. Thus, many signs from above the water lack context and are either meaningless or have a meaning which is different from that understood by terrestrial organisms.

However, despite the restrictions on sensory perception, it is significant that any individual's social interactions can add a second perceptual dimension; that of communication, social signalling, and the ability to receive and interpret social signals. Indeed, returning to the example of a fish, experiments have revealed that fish have complex cognitive and social abilities and do respond to signals from the terrestrial world (Brown 2015). As an example, salmon can use celestial navigation cues when migrating, alongside magnetic cues (Quinn 1980). Perhaps, therefore, there are untapped capabilities present in all species, which allow them to understand unfamiliar and alien phenomena, giving rise to 'semiotic freedom' (Hoffmeyer 2015). Therefore, the Umwelt can be viewed as a cognitive construct within the semiosphere. According to the definition offered by Hoffmeyer (1998), the term 'semiosphere' describes the dimension where signs occur and can be detected and/or relayed as signals. The concept of the semiosphere can thus be extended to cover all aspects of semiosis and is no longer restricted to linguistics (Kotov and Kull 2011). The semiosphere is distinct from Umwelten, because the former is made up of physical, energetic and material phenomena, including electromagnetic radiation, sound waves, mechanical forces and chemical signs, which are translated to neural signals by the sensory apparatus of organisms (Lewis, 2020). The signs and the sensory and cognitive processes form the individual's Umwelt, which gives a sign meaning to the interpreter. Clearly, part of an individual's Umwelt comprises signals from other individuals, but it is also comprised of signs in the form of inanimate objects and energy in the semiosphere. The aforementioned include geophysical formations, weather conditions, celestial objects (as are used by some migratory species to navigate, such as the salmon example given previously), magnetic fields and fluxes, chemical plumes, and landmarks such as human settlements, copses and rivers, which are also used to aid navigation (Åkesson et al. 2014). These objects are there to be detected as signs, and can become signifiers of position (Affifi 2014), even if they are not part of an individual's Umwelt 
at any given point in time, or even if an individual is not able to perceive them for a variety of reasons. Interruptions between an individual's Umwelt and the semiosphere include, for example; sensory impairment, cognitive impairment, chance occurrence and epigenetic factors.

\section{Signs, Signalling and Social Group Functioning}

By definition, social animals form groups. Even solitary and primitive species interact with other organisms; for example, sponges form symbiotic associations with bacteria (Lee et al. 2001) and termites form symbiotic associations with protozoa (Brugerolle and Radek 2006). Within a group, or indeed as part of any social interaction, deliberate communication is one identified yet complex form of signalling by which information is transferred from one individual to another (Endler 1993). This process can be interor intra-specific, and characteristically, it can be inferred to be contemporaneous, as most definitions involve the signal changing the behaviour of the receiver (Bradbury and Vehrencamp 1998); that is to say, even if a message is not instantaneously received, it should reach its receiver within a relatively short space of time, otherwise there would be no benefit to communicating.

Cultural transmissions, however, involve information, such as language, birdsong, and tool use, being transferred on a population level. This process occurs between, as well as across, multiple generations, and in each successive generation, the information is assimilated into the knowledge- base of the entire group or population (Krützen et al. 2005; Biro et al. 2003; Jordan and Shennan 2003). Thus, both communication and cultural transmission involve sign processes, and both alter the Umwelten of the individuals who are part of the sign process. Indeed, within the animal kingdom, social transmission of data means that individuals do not have to rely on personal information alone. Direct communication or signalling, eavesdropping on the signals of others (Halfwerk et al. 2014), and observations of other individual's behaviour are all forms of signs, and all mean that even in non-social species, a solipsistic cognitive schema is not realistic. An organism's semiotic scaffold is thus augmented by others, and not just its own experiences and perceptions.

Indeed, with respect human psycho-social perception, inter-connectedness has become even more complex. Technological advances mean that data can be transferred from one geographical region and culture to another, very quickly and over long ranges. Advances in travel capabilities, which include space flight, have meant that individuals can explore novel environments. Different environments can also be experienced remotely, albeit with restricted perceptual dimensions, via the internet, television, and other forms of communication technology. Further, human movement facilitates the population of new environments by invasive species, prominent examples being the muntjac deer (Stokes et al. 2006) and signal crayfish (Bubb et al. 2004). Thus, the biosemiotic constraints applied by dispersal are no longer necessarily applicable. In turn, this means that the Umwelten of many individuals have become more complex. Signs and signals now travel globally, in a planet- wide semiosphere. A wider variety of data has become available, from which information about the physical world can be gained. Indeed, human technology such as high-powered telescopes, can now detect energetic phenomena in outer- space. 
Nonetheless, in the animal kingdom, the reliability of signals as representations of the physical world can be called into question and false signalling can propagate information that is not reliable. Some animal species signal dishonestly (Ręk and Magrath, 2017; Backwell et al. 2000), and higher primates are a taxonomic group known to deliberately lie (Woodruff, and Premack 1979). However, what happens if the Umwelten of a social group are treated as a whole phenomenon, made up of discrete parts? How does the spreading of false information affect individuals within that group?

In answer, the idea of the 'Total Umwelten' (Tønnessen 2003) is applicable. It describes the sum of individual Umwelten within a population, and thus is a concept whereby each individual can share access to 'meanings' in the Umwelten of other conspecifics, or even other species (Tønnessen 2009). By taking this approach, it can be predicted that false information will have a deterministic effect, which will affect the whole group. Thus, short term gains can easily turn into long term deleterious consequences for an entire population if the information being shared is not reliable or accurate. Consequently, the meaning of signs and signals, and the physical phenomena which form a sign are interlinked, and equally as important as each other. Not only that, but ultimately, successful group functioning relies on the information being transmitted to be reliable. It also needs to be shared fairly and equitably amongst its members, whether information is passed from individual to individual via the population's genetic code, or its culture. Spreading false information (effectively a form of cheating) may benefit one individual initially, but if this behaviour disadvantages other group members, then the net effect will ultimately be disadvantageous for all of the population (Zhou and Zhang 2006). Employing game theory, cheating or deception would be unlikely to become an Evolutionarily Stable Strategy (Riehl and Frederickson 2016). Moreover, the sharing of information relies on a common understanding, or shared meaning of the signal being transmitted; essentially, a form of co-operation. This is because shared meaning relies on each individual in the group having the same understanding of a sign, or perhaps more importantly, developing and maintaining a social signal which describes and communicates a sign that can be reliably understood by group members (Jaroš 2017). Furthermore, the idea of a 'Total Umwelt' can be used to understand and predict how emergent group behaviours occur as a result of social signalling, as will be discussed subsequently.

\section{Semiosis, Shared Meaning and Emergent Group Properties}

Living organisms are made up of an assemblage cells, which have specific functions and communicate with each other to form a cohesive whole organism. Each cell transmits and receives signals, either about internal physiological processes or sensory perception of the external environment (Bruni 2008). For example, electrical nerve signals, or chemical signals such as endocrine secretions, transmit internal data related to homeostasis and structural integrity. Moreover, the sensory channels, such as the acoustic or optical channel, produce signals which can be processed internally, and this data includes information received from other organisms. Similarly, on the next semiotic level, individual organisms coexist, and social species live as part of social groups, which then form populations. In turn, populations are part of ecological 
communities. In some taxonomic groups, there is a division of labour within a social group, for example as can be seen in the social insects. In other taxa, there is no division of labour in the strictest sense, but there is evidence that personality traits can dictate the position and role of an individual in the social group (Aplin et al. 2013). Furthermore, mixed species groups have been observed to occur naturally, whereby more than one taxonomic group form symbiotic flocks, shoals or herds (Stensland et al. 2003; Ward et al. 2002; Thompson and Barnard 1984).

There are many identified means by which these groups process data and maintain social cohesion, including social bonding (Nagasawa et al. 2015; Tyack 2008), parentjuvenile attachments (Colonnello et al. 2011; Bretherton 1992), and coalitions (Detto et al. 2010; Smith et al. 2010) the latter being based upon co-operation alone. However, fundamentally, group cohesion is occurring on a semiotic level and the only change is the mode of transmission of signals, whether it be emotional or cognitive, or a combination of both. Furthermore, the process is layered, with sign processes at a cellular level, a population level, and a community level occurring laterally between individual agents on one level, and vertically, between agents on different levels. Examples of the latter are the chemical signs produced as a result of cellular physiological processes, such as changes in respiration rate which alter carbon dioxide emissions on exhalation, or immune response to infection. Indeed, it is fascinating that domestic dogs have been found to have the capability to detect disease processes in humans, including cancer and diabetes, using their olfactory sense (Wells 2012; Moser and McCulloch 2010). In this example, signs produced by a human's cellular physiology are detected by another individual of a different species via complex sign processes, not only across organisational levels, but between individuals of different species.

Therefore, to investigate group cohesion, the basic assumption needs to be that living organisms inhabit an environment made up of patterned signals. Moreover, not only do they inhabit that environment, but they are an intrinsic part of it. Living organisms receive, process and convert energy to matter and vice versa, as well as transforming energy into different forms, such as heat energy, kinetic energy and in the case of bioluminescent organisms, light energy. Each of these energy forms has its own distinct pattern; thus, the perceived world is a mixture of measurable physical phenomena and subjective experience based upon individual perceptions. A comprehensive approach, incorporating empirical data from measures of physical phenomena, and qualitative subjective analysis is therefore necessary to gain a full understanding of the living world. This is because, whilst signs clearly exist in the form of energy or matter, it is living organisms which give those signs meaning. However, significantly, the signals exist, irrespective of whether an observer perceives them or gives them meaning. Thus, the distinction between the semiosphere and Umwelten is made, because (according to traditional definitions of communication), a decision to disregard a sign or signal constitutes a change in an individual's behaviour. Nonetheless, shared meaning between different Umwelten does occur, and the semiosphere is the vehicle.

An example of a sign's semantic and pragmatic meaning can be demonstrated by vocal alarm calls, which rely on the production of sound. This involves molecules in the atmosphere being compressed and then decompressed rhythmically to form a sound wave, which travels through space and time. This is a demonstrable physical phenomenon, involving matter and energy. Nonetheless, to the receiver of the social signal, it 
forewarns danger- most likely, the approach of a predator. Furthermore, in a group living species, the behaviour of the group after the signal has been received by its members is significant. Evolutionarily, according to the Modern Synthesis, social animals live in groups because of the fitness benefits conferred, including defence against predators and group protection (Dungan et al. 2016; Parrish and EdelsteinKeshet 1999). One good example of this is group mobbing behaviour in response to an alarm call. Mobbing vocalisations are distinct calls which not only warn of danger, but 'invite' other individuals to act together to defend the group. When the signal is received, individual members of the group flock together in a co-ordinated attack on the invader. Vocalisations continue, in an effort to dissuade the predator from further attack. Whilst one individual has little chance of stopping a predatory attack, many coordinated individuals can launch a successful counterattack which protects each individual in the group (Graw and Manser, 2007; Krams and Krama 2002; McLean et al., 1986). Thus, the mobbing behaviour is an 'emergent property' of group functioning, or more specifically, of the signal's function, the sign being an approaching predator.

However, the phenomenon of co-ordinated group behaviour is not limited to group protection. For example, the 'Many Wrongs' hypothesis applies to migratory behaviour, and predicts that a group migrates by summing all of the group's data on position and direction, creating a cognitive map which is 'less wrong' than the maps of each individual group member (Codling et al. 2007). A 'murmuration' of starling's forms when individuals of the flock move in synchrony to form complex patters during flight, the purpose of which has not yet been elicited. Nonetheless, group benefits such as antipredator behaviour and attracting individuals to a roost for extra warmth in the winter, are the basis for current hypotheses (Goodenough et al. 2017). Sardine runs off the coast of South Africa, when shoals of sardines perform seamlessly synchronized movements, result in the cohesion of large groups of migrating individuals (Misund et al. 2003). Moreover, one of the most recognisable emergent behaviours is nest building in social insects, where colonies build large, complex structures in which to shelter, reproduce and store food (Theraulaz et al., 2003). In all cases, the Umwelten of individual members meet to produce an emergent group effect, which is greater than the effects produced by individual members. Whilst a lot of these behaviours rely on individuals being directed cognitively by a simple set of behavioural 'rules' (Franks et al. 1992), social signalling, such as pheromone trails in ant colonies (Attygalle and Morgan 1985) is an integral part of group cohesion and co-ordination. Indeed, performing behaviours according to cognitive rules would have little value if performed in isolation, when building a nest.

Emotionally, empathy is also a means by which Umwelten merge, and individuals of many species can experience the emotions and feelings of another individual via signs such as facial expression, kinesics and vocal signalling. Thus, empathy is a means by which social bonds can occur (Clay and de Waal 2013; Romero et al. 2013). Further, in human psychology, more complex emergent behaviours, such as projective identification, can be observed. This involves a subject 'projecting' their own undesirable characteristics onto an object who 'accepts' the projection, and in return relinquishes their own more positive characteristics to the subject (Tansey and Burke 2013; Ogden 1979). Both agents swap roles almost involuntarily, as a result of social interactions, empathy, and psychological manipulation, to produce an effect which can alter group dynamics as well as inter-personal interactions. 
Significantly, biosemiotics and Umwelt theory provide the unifying factor for all these examples within the fields of ethology, psychology and behavioural ecology, and the basic principal is described in fig. 1. The Umwelten of individuals remain distinct phenomena, but there is overlap within the semiosphere, and it is at the point of overlap that emergent properties occur.

By viewing sign processes and information transfer as being the basic unit of a group, a framework from which to study these 'emergent' phenomena becomes evident. Semiotically, the Umwelten of each individual group member can be seen to be joining to form one whole, analogous to an individual's co-ordinated cellular immune response to infection, or, indeed, a neural network which produces consciousness. Thus, the perceptual information of each individual is pooled, and any deficits of individual group members are compensated for by the group as a whole. Moreover, whilst population and community interactions are occurring, these sign processes are, in turn, the result of energetic and chemical processes at a cellular level within individual organisms. Communications between neurons, and somatic cells elicit cognitive processes and behaviours in individuals within groups, which in turn drive the formation of what amount to meta- neural networks. Indeed, this 'connectedness' can be seen in the organisational structure of the colonial Siphonophorae, which are communities of individual polyps in which physiological functions, such as feeding and reproduction, are divided amongst specialized polypoid group members. Co-

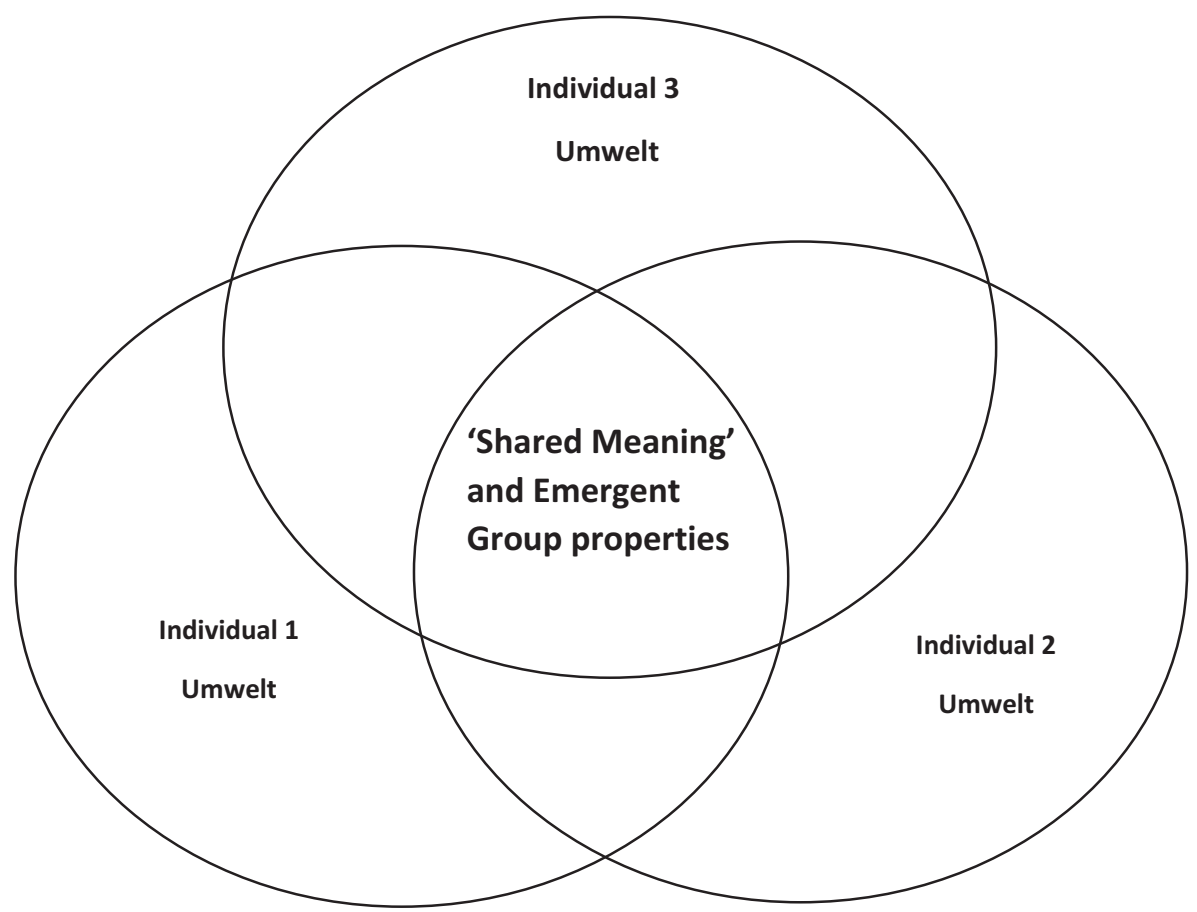

Fig. 1 Overlapping Umwelten where shared meaning gives rise to emergent group behavioural properties at the intersect. Shared meaning occurs at each intersect, but it is only the middle intersect where all group members can comprehend each other's meaning of signs and social signals. This is where, in the semiosphere, emergent properties of the group are predicted to occur 
existing symbiotically, the colonial Siphonophorae, such as the Portuguese man o'war (Physalia physalis), are actually many individuals functioning collectively as one whole organism (Hessinger and Ford 2012). In this example, the resemblance to a matrix of somatic cells, or a neural network of neurons, but on a larger scale, is striking, as is the similarity to a free- living social group. Further, it is a striking feature of mammalian species that the neurohormonal signalling involved in physical bodily damage is the same sign process which is engaged when a social bond is lost. Significantly, the sensation described is 'pain', suggesting that individuals in a group respond to 'damage' to the social group, or loss of a socially bonded individual, in the same way an individual responds to cellular tissue damage (Eisenberger 2012; MacDonald and Leary 2005).

\section{Conclusion}

From a cellular level, to a whole organism level, to a population and community level, living organisms form complex layers and webs of sign processes. Group organisation relies on social signalling and the sharing of information gleaned from signs. Even solitary living animals must rely on other organism's signs to some degree; for example, a predatory species must learn to understand signs from its prey to execute a successful capture. Similarly, prey species need to be able to detect and predict predatory behaviour to attempt escape. Further, eavesdropping, for example on alarm calls, relies on intraspecific meeting of Umwelten so that meaning is shared across taxa. Moreover, some species live co-operatively in mixed species groups, and the meanings of signs are shared across species on a social level. One of the most striking examples of intraspecific 'shared meaning' is the keeping of companion animals in human societies. Indeed, by understanding and manipulating signs and sign processes on both a genetic and social level, humans have managed to domesticate a potentially very dangerous apex predator; the wolf. The domestic dog, Canis lupus familiaris, a descendant of wild wolves, lives with humans in their homes and social groups. Dogs contribute to work related tasks such as herding, guarding and retrieving; sporting activities, such as obedience and agility, and provide companionship and social interaction for their human group members, and vice versa. Whilst the balance of power and control is biased toward the human agents, the domestic dog lives symbiotically, benefiting from shelter, food, veterinary care, protection and social interaction.

Thus, the most promising approach to studying social behaviour may be to view social groups as functioning as a whole organism, with shared meaning in the groups' interpretation of the environment, as it acts to process energy (forage), reproduce, and migrate collectively; indeed, in the same way that an individual multicellular organism functions on a cellular level. The 'Total Umwelt' and shared meaning gives significant scope to explore this concept further.

Acknowledgments With thanks to Sebastian Lewis for his help in understanding the concept of the Umwelt. Many thanks also to the anonymous reviewers, whose invaluable comments, discussion and guidance helped develop and shape this article. Their assistance with terminology is also appreciated. 
Open Access This article is licensed under a Creative Commons Attribution 4.0 International License, which permits use, sharing, adaptation, distribution and reproduction in any medium or format, as long as you give appropriate credit to the original author(s) and the source, provide a link to the Creative Commons licence, and indicate if changes were made. The images or other third party material in this article are included in the article's Creative Commons licence, unless indicated otherwise in a credit line to the material. If material is not included in the article's Creative Commons licence and your intended use is not permitted by statutory regulation or exceeds the permitted use, you will need to obtain permission directly from the copyright holder. To view a copy of this licence, visit http://creativecommons.org/licenses/by/4.0/.

\section{References}

Affifi, R. (2014). Biological pedagogy as concern for semiotic growth. Biosemiotics, 7(1), 73-88.

Åkesson, S., Boström, J., Liedvogel, M., \& Muheim, R. (2014). Animal navigation. Animal movement across scales, 21, 151-178.

Aplin, L., Farine, D., Mornad-Ferron, J., Cole, E., Cockburn, A., \& Sheldon, B. (2013). Individual persoanlities predict social behaviour in wild networks of great tits (Parus major). Ecology Letters, 16, 12181.

Attygalle, A. B. and Morgan, E. D. (1985). Ant trail pheromones. In Advances in insect physiology (Vol. 18, pp. 1-30). Cambridge, MA, USA: Academic press.

Backwell, P. R., Christy, J. H., Telford, S. R., Jennions, M. D., \& Passmore, J. (2000). Dishonest signalling in a fiddler crab. Proceedings of the Royal Society of London. Series B: Biological Sciences, 267(1444), 719-724.

Biro, D., Inoue-Nakamura, N., Tonooka, R., Yamakoshi, G., Sousa, C., \& Matsuzawa, T. (2003). Cultural innovation and transmission of tool use in wild chimpanzees: Evidence from field experiments. Animal Cognition, 6(4), 213-223.

Bradbury, J. W., \& Vehrencamp, S. L. (1998). Principles of animal communication. 349-350: Sunderland MA, USA: Sinauer Associates.

Bretherton, I. (1992). The origins of attachment theory: John Bowlby and Mary Ainsworth. Developmental Psychology, 28(5), 759-775.

Brown, C. (2015). Fish intelligence, sentience and ethics. Animal Cognition, 18(1), 1-17.

Brugerolle G., Radek R. (2006) Symbiotic Protozoa of termites. In: König H., Varma a. (Ed.) Intestinal Microorganisms of Termites and Other Invertebrates. Soil Biology, vol 6. Springer, Berlin, Heidelberg.

Bruni, L. E. (2008). Cellular semiotics and signal transduction. In M. Barbieri (Ed.), Introduction to biosemiotics (pp. 365-408). Dordrecht: Springer.

Bubb, D. H., Thom, T. J., \& Lucas, M. C. (2004). Movement and dispersal of the invasive signal crayfish Pacifastacus leniusculus in upland rivers. Freshwater Biology, 49(3), 357-368.

Clay, Z., de Waal, F.B., (2013). Development of socio-emotional competence in bonobos. Proceedings of the National Academy of Sciences, 201316449.

Codling, E. A., Pitchford, J. W., \& Simpson, S. D. (2007). Group navigation and the "many-wrongs principle" in models of animal movement. Ecology, 88(7), 1864-1870.

Colonnello, V., Iacobucci, P., Fuchs, T., Newberry, R. C., \& Panksepp, J. (2011). Octodon degus. A useful animal model for social-affective neuroscience research: Basic description of separation distress, social attachments and play. Neuroscience \& Biobehavioral Reviews, 35(9), 1854-1863.

Detto, T., Jennions, M. D., \& Backwell, P. R. (2010). When and why do territorial coalitions occur? Experimental evidence from a fiddler crab. The American Naturalist, 175(5), E119-E125.

Dungan, S. Z., Wang, J. Y., Araújo, C. C., Yang, S. C., \& White, B. N. (2016). Social structure in a critically endangered indo-Pacific humpback dolphin (Sousa chinensis) population. Aquatic Conservation: Marine and Freshwater Ecosystems, 26(3), 517-529.

Eisenberger, N. I. (2012). The pain of social disconnection: Examining the shared neural underpinnings of physical and social pain. Nature Reviews Neuroscience, 13(6), 421-434.

Endler, J. A. (1993). Some general comments on the evolution and design of animal communication systems. Philosophical Transactions of the Royal Society of London Series B: Biological Sciences, 340(1292), 215-225.

Franks, N. R., Wilby, A., Silverman, B. W., \& Tofts, C. (1992). Self-organizing nest construction in ants: Sophisticated building by blind bulldozing. Animal Behaviour, 44, 357-375. 
Ginn, F. (2014). Jakob von Uexküll beyond bubbles: On Umwelt and biophilosophy. Science as Culture, 23(1), 129-134.

Goodenough, A. E., Little, N., Carpenter, W. S., \& Hart, A. G. (2017). Birds of a feather flock together: Insights into starling murmuration behaviour revealed using citizen science. PLoS One, 12(6), e0179277.

Graw, B., \& Manser, M. B. (2007). The function of mobbing in cooperative meerkats. Animal Behaviour, 74(3), 507-517.

Halfwerk, W., Jones, P. L., Taylor, R. C., Ryan, M. J., \& Page, R. A. (2014). Risky ripples allow bats and frogs to eavesdrop on a multisensory sexual display. Science, 343(6169), 413-416.

Hessinger, D. A., \& Ford, M. T. (2012). Of the Portuguese man-of-war (Physalia physalis) tentacle. The Biology of Nematocysts, 75.

Hoffmeyer, J. (1998). Surfaces inside surfaces. On the origin of agency and life. Cybernetics \& Human Knowing, 5(1), 33-42.

Hoffmeyer, J. (2015). Semiotic scaffolding: A unitary principle gluing life and culture together. Green Letters, $19(3), 243-254$.

Hoffmeyer, J. (2008a). The semiotic niche. Journal of Mediterranean Ecology, 9, 5-30.

Hoffmeyer, J. (2008b). Semiotic scaffolding of living systems. In M. Barbieri (Ed.), Introduction to biosemiotics. Dordrecht: Springer.

Jaroš, F. (2017). The three semiotic lives of the domestic cat: A case study on animal social cognition. Biosemiotics, 10(2), 279-293.

Jordan, P., \& Shennan, S. (2003). Cultural transmission, language, and basketry traditions amongst the California Indians. Journal of Anthropological Archaeology, 22(1), 42-74.

Kotov, K., \& Kull, K. (2011). Semiosphere is the relational biosphere. In Towards a semiotic biology: Life is the action of signs (pp. 179-194). London: Imperial College Press.

Krützen, M., Mann, J., Heithaus, M. R., Connor, R. C., Bejder, L., \& Sherwin, W. B. (2005). Cultural transmission of tool use in bottlenose dolphins. Proceedings of the National Academy of Sciences, 102(25), 8939-8943.

Krams, I., \& Krama, T. (2002). Interspecific reciprocity explains mobbing behaviour of the breeding chaffinches, Fringilla coelebs. Proceedings of the Royal Society of London. Series B: Biological Sciences, 269(1507), 2345-2350.

Lee, Y. K., Lee, J. H., \& Lee, H. K. (2001). Microbial symbiosis in marine sponges. Journal of MicrobiologySeoul, 39(4), 254-264.

Lewis, A. (2020). In search of a unified theory of sensory perception: Possible links between the vibrational mechanism of olfaction and the evolution of language. Biosemiotics, 1-10.

MacDonald, G., \& Leary, M. R. (2005). Why does social exclusion hurt? The relationship between social and physical pain. Psychological Bulletin, 131(2), 202-223.

McLean, I. G., Stewart, K. G., \& Smith, J. N. (1986). Mobbing behaviour, nest exposure, and breeding success in the American Robin. Behaviour, 96(1-2), 171-185.

Moser, E., \& McCulloch, M. (2010). Canine scent detection of human cancers: A review of methods and accuracy. Journal of Veterinary Behavior, 5(3), 145-152.

Nagasawa, M., Mitsui, S., En, S., Ohtani, N., Ohta, M., Sakuma, Y., Onaka, T., Mogi, K., \& Kikusui, T. (2015). Oxytocin-gaze positive loop and the coevolution of human-dog bonds. Science, 348(6232), 333336.

Parrish, J. K., \& Edelstein-Keshet, L. (1999). Complexity, pattern, and evolutionary trade-offs in animal aggregation. Science, 284(5411), 99-101.

Misund, O. A., Coetzee, J. C., Fréon, P., Gardener, M., Olsen, K., Svellingen, I., \& Hampton, I. (2003). Schooling behaviour of sardine Sardinops sagax in false bay, South Africa. African Journal of Marine Science, 25, 185-193.

Ogden, T. H. (1979). On projective identification. International Journal of Psycho-Analysis, 60, 357-373.

Quinn, T. P. (1980). Evidence for celestial and magnetic compass orientation in lake migrating sockeye salmon fry. Journal of Comparative Physiology, 137(3), 243-248.

Ręk, P., \& Magrath, R. D. (2017). Deceptive vocal duets and multimodal display in a songbird. Proceedings of the Royal Society B: Biological Sciences, 284(1864), 20171774.

Riehl, C., \& Frederickson, M. E. (2016). Cheating and punishment in cooperative animal societies. Philosophical Transactions of the Royal Society B: Biological Sciences, 371(1687), 20150090.

Romero, T., Konno, A., \& Hasegawa, T. (2013). Familiarity bias and physiological responses in contagious yawning by dogs support link to empathy. PLoS One, 8(8).

Smith, J. E., Van Horn, R. C., Powning, K. S., Cole, A. R., Graham, K. E., Memenis, S. K., \& Holekamp, K. E. (2010). Evolutionary forces favoring intragroup coalitions among spotted hyenas and other animals. Behavioral Ecology, 21(2), 284-303. 
Stensland, E. V. A., Angerbjörn, A., \& Berggren, P. E. R. (2003). Mixed species groups in mammals. Mammal Review, 33(3-4), 205-223.

Stokes, K. E., Montgomery, W. I., Dick, J. T. A., Maggs, C. A., \& McDonald, R. A. (2006). The importance of stakeholder engagement in invasive species management: A cross-jurisdictional perspective in Ireland. Biodiversity and Conservation, 15(8), 2829-2852.

Tansey, M. J., \& Burke, W. F. (2013). Understanding countertransference: From projective identification to empathy. London: Routledge.

Theraulaz, G., Gautrais, J., Camazine, S., \& Deneubourg, J. L. (2003). The formation of spatial patterns in social insects: From simple behaviours to complex structures. Philosophical Transactions of the Royal Society of London. Series A: Mathematical, Physical and Engineering Sciences, 361(1807), 1263-1282.

Thompson, D. B. A., \& Barnard, C. J. (1984). Prey selection by plovers: Optimal foraging in mixed-species groups. Animal Behaviour, 32(2), 554-563.

Tønnessen, M. (2009). Abstraction, cruelty and other aspects of animal play (exemplified by the playfulness of Muki and Maluca). Sign System Studies, 37(3/4), 558-579.

Tønnessen, M. (2003). Umwelt ethics. Sign Systems Studies, 31(1), 281-299.

Tyack, P. L. (2008). Convergence of calls as animals form social bonds, active compensation for noisy communication channels, and the evolution of vocal learning in mammals. Journal of Comparative Psychology, 122(3), 319-331.

Ward, A. J., Axford, S., \& Krause, J. (2002). Mixed-species shoaling in fish: The sensory mechanisms and costs of shoal choice. Behavioral Ecology and Sociobiology, 52(3), 182-187.

Wells, D. L. (2012). Dogs as a diagnostic tool for ill health in humans. Alternative Therapies in Health \& Medicine, 18(2).

Woodruff, G., \& Premack, D. (1979). Intentional communication in the chimpanzee: The development of deception. Cognition, 7(4), 333-362.

Zhou, L., \& Zhang, D. (2006). A comparison of deception behavior in dyad and triadic group decision making in synchronous computer-mediated communication. Small Group Research, 37(2), 140-164.

Publisher's Note Springer Nature remains neutral with regard to jurisdictional claims in published maps and institutional affiliations. 